\title{
Evaluation of Biological Activity of Different Wavelengths of Low-Level Laser Therapy on the Cancer Prostate Cell Line Compared With Cisplatin
}

\author{
Jaber Zafari $^{\circledR}$, Hossein Abbasinia $^{\circledR}$, Hediyeh Gharehyazi ${ }^{\circledR}$, Fatemeh Javani Jouni ${ }^{(}$, Saeed Jamali $^{(\mathbb{D}}$, \\ Mohammadreza Razzaghi ${ }^{* \text { (D) }}$
}

${ }^{1}$ Laser Application in Medical Sciences Research Center, Shahid Beheshti University of Medical Sciences, Tehran, Iran ${ }^{2}$ Department of Cellular and Molecular Biology, Faculty of Advanced Sciences and Technology, Tehran Medical Sciences, Islamic Azad University, Tehran, Iran

${ }^{3}$ Department of Biomedical Engineering, Faculty of Health, Tehran Medical Sciences, Islamic Azad University, Tehran, Iran

\section{*Correspondence to \\ Mohammadreza Razzaghi, Laser Application in Medical Sciences Research Center, Shahid Beheshti University of Medical Sciences, Tehran, Iran; Tel: +98-2122718021; \\ Email: m-razaghi@sbmu.ac.ir}

Published online May 1, 2021

\begin{abstract}
Introduction: Cancer is one of the most important problems in the world. Low-level laser therapy (LLLT) has been emerged as a new approach, having both stimulation and inhibition effects on cellular function. The goal of this study was to analyze and compare the different concentrations of cisplatin and wavelengths of laser therapy on the LnCap cell lines.

Methods: LnCap cells were cultured and treated with different concentrations of cisplatin $(0.1,0.4$, $0.8,1.2$ and $2 \mu \mathrm{g} / \mathrm{mL}$ for 24 hours) and wavelengths of laser therapy $(610,630$ and $810 \mathrm{~nm})(0.45$ $\mathrm{J} / \mathrm{cm}^{2}$ ) separately. The viability of cells was examined by MTT assay and IC50 was also calculated. Furthermore, a combination of cisplatin IC50 (24 hours) and different wavelengths of the laser was examined.

Results: The results of this study showed that $2 \mu \mathrm{g} / \mathrm{mL}$ of cisplatin has the most significant reduction effect on the cell viability of the LnCap cell line. Cisplatin decreased the viability of cells in a dosedependent manner. Moreover, IC50 of cisplatin was $1.24 \mu \mathrm{g} / \mathrm{mL}$. On the other hand, LLLT with wavelengths of 610,630 and $810 \mathrm{~nm}$ did not show notable biological effects on cell viability.

Conclusion: As known, cisplatin has the capability to reduce the viability of LnCap cell lines. However, LLLT cannot be a remarkable option for the treatment of prostate cancer. Therefore, although laser therapy showed praiseful therapeutic activity against some cancer cell lines, in this study the results indicated that defined laser wavelengths had no inhibitory effects against the prostate cancer cell line.

Keywords: Low-Level Laser Therapy; Chemotherapy; Cisplatin; Prostate Cancer
\end{abstract}

\section{Introduction}

Cancer is one of the most common health concerns in the world and the second cause of death in the United States. Among all cancer types, prostate cancer is one of the major men's problems ${ }^{1}$. Normal prostate tissue relies on androgen epithelial secretory activity so prostate tissue function and development depend on androgen continuity. After the binding of testosterone, dihydrotestosterone binds to the androgen receptor (AR) and then AR translocates into the nucleus and regulates the transcription of target genes. Epithelial cell proliferation and apoptosis are regulated by AR signaling. Prostate cancer develops as a result of imbalanced AR signaling. ${ }^{2}$ Prostate Cancer is one of the most important problems in the world and it was estimated that 191.930 new cases of prostate cancer and 33.330 deaths occurred in the United States in $2020 .{ }^{1}$ Chemotherapy and radiation therapy are effective strategies against many types of cancers; however, they might kill or damage normal cells and lead to side effects such as fatigue, hair loss, coagulation problems, lymph node edema, and anemia. Due to these problems, the application of high doses of anti-cancer drugs is quite impossible. ${ }^{3}$ Traditional methods have severe side effects with some resistance mechanisms against chemotherapy, including increased drug efflux, mutations of the drug targets, DNA damage repair, evasion of cell death and activation of alternative signaling pathways. ${ }^{4}$ Cisplatin, one of the most important solid tumor drugs, has been used in cancer treatment for more than 30 years. It interacts with the DNA strand and is considered as an important factor in cell cycle arrest and the induction of cancer cell apoptosis. ${ }^{5}$ However, cancer cells can be resistant to cisplatin through mechanisms including changes in cellular uptake, increased detoxification,

Please cite this article as follows: Zafari J, Abbasinia H, Gharehyazi H, Javani Jouni F, Jamali S, Razzaghi M. Evaluation of biological activity of different wavelengths of low-level laser therapy on the cancer prostate cell line compared with cisplatin. J Lasers Med Sci. 2021;12:e17. doi:10.34172/jlms.2021.17 
drug efflux, increased DNA repair and inhibition of apoptosis. ${ }^{6,7}$ Low-level laser therapy (LLLT) is a new method that is reported as a promising treatment for reducing pain, inflammation, edema, and promotion of damaged tissue healing. ${ }^{8}$ LLLT exerts its impacts through cellular mechanisms such as changing the production procedure of ATP, nitric oxide (NO), reactive oxygen species (ROS) and gene transcription/expression. ${ }^{8}$ It has been shown that LLLT may be regarded as a treatment approach in breast cancer. ${ }^{9}$ Hence, due to the limited studies in this field, there is a crucial need for more clinical researches. ${ }^{9}$ Laser focal therapy is a new less invasive method for prostate cancer therapy, being based on heating the prostate tissue with an infrared laser in order to cause tumor destruction. ${ }^{10}$ Therefore, physical agents such as laser therapy can be useful because of their non-invasive effects and lower medical costs. ${ }^{11}$ This study aims to understand the therapeutic score of different wavelengths of laser therapy (610,630 and $810 \mathrm{~nm})$ as a therapeutic method in a complementary approach to common guidelines and comparative analysis with cisplatin as a common therapeutic agent in the prostate cancer cell lines (LnCap).

\section{Material and Methods Cell Culture}

The LnCap prostate cancer cell line was obtained from the National Cell Bank of Iran (NCBI). The cells were cultured in Dulbecco's Modified Eagle medium (DMEM) (GIBCO) including fetal bovine serum (10\% FBS) (GIBCO, Life Technologies, Ghent, Belgium), $100 \mu \mathrm{g} / \mathrm{mL}$ streptomycin and $100 \mathrm{U} / \mathrm{mL}$ of penicillin/streptomycin. The cells were kept at $37^{\circ} \mathrm{C}$ and $5 \% \mathrm{CO}_{2}$ in the incubator in order to reach $70 \%-80 \%$ confluency.

\section{Cisplatin and Laser Treatment}

To investigate the effect of cisplatin and the laser on the viability of cells, we established four main groups. The cells in the first group were treated with different wavelengths of the laser $(610,630$ and $810 \mathrm{~nm})$. The laser probe was held perpendicular to each plate and the cells were irradiated from underneath each well from a $5 \mathrm{~mm}$ distance. The characteristics of different doses of laser wavelengths have been arranged in Table 1.

The cells of the second group were treated with different concentrations of cisplatin $(0.1,0.4,0.8,1.2$ and $2 \mu \mathrm{g} / \mathrm{mL})$ for 24 hours. The cells of the third group were treated with IC50 (24 hours) of cisplatin and different wavelengths of the laser $(610,630$ and $810 \mathrm{~nm})\left(0.45 \mathrm{~J} / \mathrm{cm}^{2}\right)$. The fourth group was considered as the control group (the group with no treatment).

\section{MTT Assay}

Confluent LnCap cells were seeded in a 96-well plate and the MTT assay for all cisplatin, the laser, cisplatin/ different wavelengths of the laser and control groups was
Table 1. The Characteristics of Different Doses of Laser Wavelengths

\begin{tabular}{lcc}
\hline Name Company Laser & Wavelength $(\mathbf{n m})$ & Dose $\left(\mathbf{J} / \mathbf{c m}^{2}\right)$ \\
\hline Ploon & 610 & 0.45 \\
Ploon & 630 & 0.45 \\
Elexxion nano & 810 & 0.45 \\
\hline
\end{tabular}

carried out. The cells were seeded into a 96-well plate (5$6 \times 10^{3}$ per well) and the respected wells were incubated for 24 hours. In the next step, the medium was removed and each well was treated with different concentrations of cisplatin $(0.1,0.4,0.8,1.2$ and $2 \mu \mathrm{g} / \mathrm{mL})$ for 24 hours. One hundred microliters of $500 \mu \mathrm{g} / \mathrm{mL} 3-(4,5$ dimethylthiazol2-yl)-2,5-diphenyltetrazolium bromide (MTT) was added and the cells were incubated again for 4 hours. After that, the supernatant of the cells was aspirated off and $50 \mu \mathrm{L}$ of DMSO was added. Finally, the BioTek ELx808 microplate reader was used to analyze the absorbance of the cells at $570 \mathrm{~nm}$. Cell viability was reported as a percentage and compared with the control group. By the means of MTT assay, the half maximal inhibitory concentration (IC50) of cisplatin was also calculated.

\section{Statistical Analysis}

The data were reported as mean \pm standard deviation (SD) and analyzed by one-way or two-way analysis of variance (ANOVA). Results with $P$ value less than 0.05 were considered to be significant. Dose-response curves for defining inhibitory concentration (IC50) values were depicted by GraphPad Prism 5 and Compusyn software. The error bars in the results came from 5 experimental repetitions.

\section{Results}

\section{MTT Analysis}

To examine the effect of different wavelengths of the laser and cisplatin on the cell viability, LnCap cells were treated with wavelengths of 610,630 and $810 \mathrm{~nm}$ of the laser (Figure 1) and $0.1,0.4,0.8,1.2$ and $2 \mu \mathrm{g} / \mathrm{mL}$ of cisplatin separately (Figure 2). As it is clear from the graph, cell viability significantly decreased in a dose-dependent manner in the cisplatin-treated group. However, the effect of different wavelengths of the laser on the cell viability of LnCap cells was not significant.

\section{IC50 Concentration of Cisplatin}

The effect of different concentrations of cisplatin (0.1, $0.4,0.8,1.2$ and $2 \mu \mathrm{g} / \mathrm{mL}$ ) on LnCap cell viability was examined and a semi-logarithmic graph of cisplatin was drawn to obtain IC50 (Figure 3). The half maximal inhibitory concentration (IC50) of cisplatin was needed to measure the $50 \%$ inhibition capacity of cisplatin toward cell growth. The calculated IC50 was found to be $1 \mathrm{mM}$ in this study, which represents the concentration of $1.24 \mu \mathrm{g} /$ $\mathrm{mL}$ for cisplatin. 


\section{Combination of Cisplatin and Different Wavelengths of the Laser}

A combination of cisplatin ic50 (24 hours) and different wavelengths of the laser is shown in Figure 4. This combination reduced the cell viability of LnCap more than cisplatin alone. However, all the applied laser wavelengths caused significant efficiency of cisplatin treatment between all groups; the effect of cisplatin elevated in the 630 and $810 \mathrm{~nm}$ wavelengths more than the $610 \mathrm{~nm}$ one.

\section{Discussion}

Dysregulation of cell division and cell cycle progression can cause cancer. Regulatory factors, stress signals and tumor environment resulting from damaged DNA are determining factors, deciding whether the cells can proliferate or die. ${ }^{12}$ Prostate cancer is one of the common types of cancer and also the third main cause of death in men in the United States. ${ }^{13}$ LLLT can modulate the inflammatory response, reduce pain and healing time and promote cellular bio-stimulation. As an example, recurrent artophagous ulcers (RAU) are the common type of ulcer in the oral cavity and LLLT has been suggested as an alternative treatment for that. In fact, there are pieces

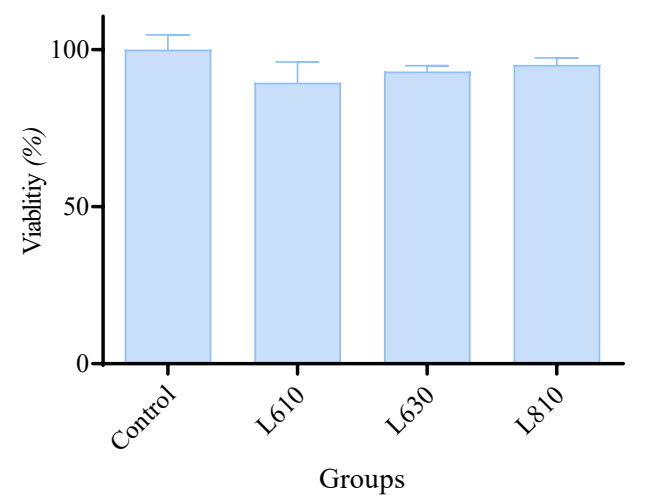

Figure 1. The Effect of Three Different Laser Wavelengths (610, 630 and $810 \mathrm{~nm})\left(0.45 \mathrm{~J} / \mathrm{cm}^{2}\right)$ on LnCap Cells.

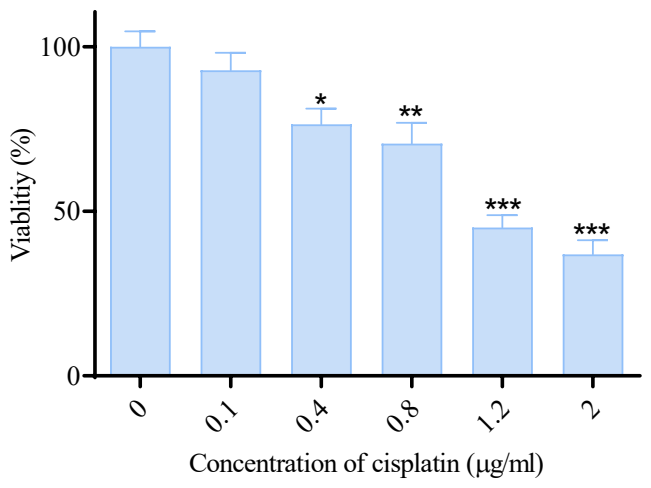

Figure 2. The Effect of Different Concentrations of Cisplatin on LnCap Cells After 24 hours. All groups were compared with the control group $\left(* P<0.5,{ }^{* *} P<0.01,{ }^{* * *} P<0.001\right)$

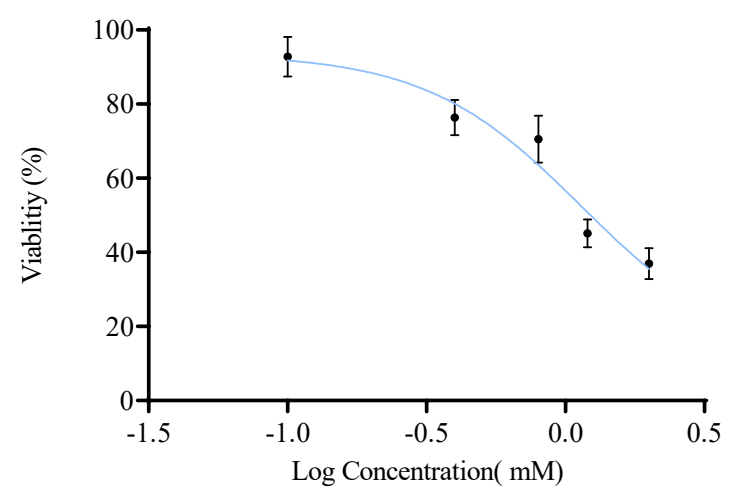

Figure 3. Semi-logarithmic Graph of Cisplatin to Obtain IC50.

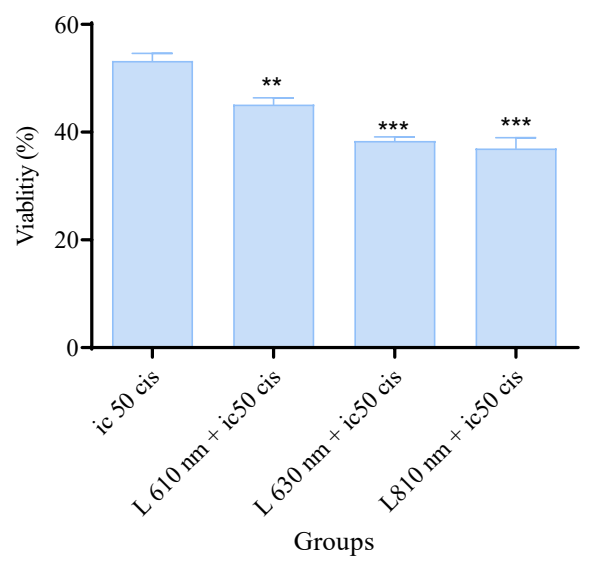

Figure 4. The Effect of IC50 (24 hours) of Cisplatin and Different Wavelengths of the Laser $(610,630$ and $810 \mathrm{~nm})\left(0.45 \mathrm{~J} / \mathrm{cm}^{2}\right)$ on LnCap Cells. All groups were compared with the control group (*** $P<0.001)$.

of clear evidence that wavelengths have an important and effective role in RAU treatment. ${ }^{14}$ In addition, laser therapy has been reported as a new choice of cancer treatment that needs more examination. ${ }^{15}$ The application of laser therapy as a non-invasive treatment of urologic diseases has attracted a high number of researchers in recent years. The less expensive, more compact, userfriendly, high power, easy to handle and convenient technique like laser therapy is attractive for clinicians, and understanding the wavelength of light which is used and interacted with living cells is important. ${ }^{16,17}$

Due to the nearly fixed chemotherapy protocol for the treatment of patients, resistance to chemotherapy is regarded as a fundamental obstacle in this way. Cisplatin is one of the important solid tumor drugs, which has been used for cancer therapy for several years. The interaction with DNA strands, inhibition and causing damage to DNA strands, arresting the cell cycle and induction of apoptosis are the mechanisms of cisplatin functions. ${ }^{5}$ Resistance to cisplatin is a major problem and increasing the drug dose to overcome this resistance is another problem which might lead to unwanted side effects. ${ }^{6,18,19}$ Decreased drug 
intake, drug efflux, alternation of drug metabolisms and the DNA repair process and also the enhancement of the number of target molecules in the cells have been reported as resistant mechanisms for cisplatin drugs. ${ }^{18}$

After the invention of the laser, it has been used for wound and nerve healing, pain soothing, and treatment of edema and inflammation for about 40 years. Cellular mechanisms have been affected by this technique and mitochondria are the initial site in this process leading to the enhancement of ATP production, the regulation of reactive oxygen species and the induction of transcription factors. Therefore, these effects can cause increased cell proliferation and migration. ${ }^{8}$

LLLT has emerged as a new approach that has both stimulatory and inhibitory effects on cellular functions. It is found that laser irradiation could increase ATP synthesis, ROS generation and NO release after cells exposure to a laser. In addition, ROS might have an important role in the signaling pathway, under the effect of LLLT. It also can induce the expression of some transcription factors like nuclear factor kappa B (NF- $\kappa \beta)$ that can affect the transcription of other genes. It has been reported that laser therapy can inhibit apoptosis and increase proliferation, addition and migration. It was shown that 30-minute exposure to red (632 $\mathrm{nm}$ or 670 $\mathrm{nm})$ or near IR $(810 \mathrm{~nm})$ can stimulate wound healing and reduce swelling. Today, worldwide researches have been conducted to evaluate the therapeutic option of LLLT in rheumatoid, Alzheimer's disease, nerve regeneration, heart attack, spinal cord injury and stroke. ${ }^{20}$

As known, LLLT induces some cellular mechanisms such as proliferation, differentiation, and even apoptosis by triggering cellular pathways and the activation of the mitochondrial respiratory chain. LLLT can enhance the release and synthesis of growth factors, inflammatory cytokines and interleukins. In this way, specific receptors that might accept the energy of a laser will increase, leading to autophosphorylation, some downstream events and also the enhancement of intracellular calcium concentration and cell proliferation. Low level laser elevate ATP/AMP ratio. After the elevation of synthesis of ATP and protein, the expression of cytokines and growth factors will increase and finally lead to cell proliferation. In fact, a laser can change cellular homeostasis and functions by altering $\mathrm{PHi}$, the cellular redox state, and enhancing the expression of redox sensitive factors such as NF- $\kappa \beta$ which induce cell proliferation. ${ }^{21}$ It is important that these effects depend strongly on the applied laser wavelength. In this study, the LnCap cell line was exposed to different wavelengths of LLLT $(610,630$ and $810 \mathrm{~nm}$ of laser wavelength) and it was shown that LLLT did not have any significant effect on the LnCap cell line at the chosen dosages for this investigation. On the other hand, the effect of cisplatin on the LnCap cell line was analyzed and IC50 $(1.24 \mu \mathrm{g} / \mathrm{mL})$ was also calculated. It was illustrated that cisplatin has the capability to decrease the cell viability of the LnCap cell line in a dose-dependent manner. In fact, cisplatin can attach to DNA, block the DNA replication and induce apoptotic signals, ${ }^{22,23}$ and it was also confirmed in our study. As it can be seen, the effects of LLLT on the viability of LnCap cells were not significant alone. Antitumor immune response, induction of inflammatory response and apoptosis of cancer cells are other impacts of this method. ${ }^{24}$ Crescenzi et al examined the combination of cisplatin and photodynamic therapy (an IR diode laser source at $805 \mathrm{~nm}$ ) and reported their synergic effects. ${ }^{25}$ De Freitas et al examined the effects of combination therapy (Photodynamic therapy and cisplatin) on siHa, HaCaT and C-33A cell lines in 2014. In this research, the cell lines were exposed to photodynamic therapy (630 or a $660 \mathrm{~nm}$ LED light source) in the initial level and treated with cisplatin in the next step. It was shown that compared to other groups, the efficiency of cisplatin increased and the viability of cells decreased in the photodynamic-cisplatin treated group. ${ }^{26}$ The applied laser wavelengths had no cytotoxic effects against the prostate cancer cell line alone; they strongly enhanced the effect of cisplatin in a combined manner.

\section{Conclusion}

According to the findings, it could be concluded that applying a combination therapy manner by using a laser with common anticancer drugs like cisplatin posed a considerable elevation in cancer therapy guidelines. Along with no demonstrated adverse effects of applied laser wavelengths, this study proposed the combination therapy of a laser with platina core cancer drugs as cisplatin in cancer therapy guidelines based on platina chemotherapy such as prostate cancer. Although the results revealed a praiseful aim in increasing the efficiency of cancer therapy guidelines, this study suggested the necessity of more investigations to examine the synergic effects of combination therapy (photodynamic therapy and cisplatin) on these cell lines.

\section{Ethical Considerations}

This study was approved by the ethics committee of Shahid Beheshti University of Medical Sciences (IR. SBMU.RETEC.REC.1399.401).

\section{Conflict of Interests}

The authors declare no conflict of interest.

\section{Reference}

1. Siegel RL, Miller KD, Jemal A. Cancer statistics, 2020. CA Cancer J Clin. 2020;70(1):7-30. doi: 10.3322/caac.21590.

2. Erdogan B, Kostek O, Bekirhacioglu M. Enzalutamide in Prostate Cancer, A Review on Enzalutamide and cancer. Eurasian J Med Oncol. 2018;2(3):121-9. doi: 10.14744/ ejmo.2018.72098.

3. Siegel R, DeSantis C, Virgo K, Stein K, Mariotto A, Smith T, et al. Cancer treatment and survivorship statistics, 2012. CA Cancer J Clin. 2012;62(4):220-41. doi: 10.3322/caac.21149.

4. Holohan C, Van Schaeybroeck S, Longley DB, Johnston 
PG. Cancer drug resistance: an evolving paradigm. Nat Rev Cancer. 2013;13(10):714-26. doi: 10.1038/nrc3599.

5. Basu A, Krishnamurthy S. Cellular responses to Cisplatininduced DNA damage. J Nucleic Acids. 2010; 2010:201367. doi: 10.4061/2010/201367.

6. Florea A-M, Büsselberg D. Cisplatin as an anti-tumor drug: cellular mechanisms of activity, drug resistance and induced side effects. Cancers. 2011;3(1):1351-71. doi: $10.3390 /$ cancers 3011351 .

7. Ghosh S. Cisplatin: The first metal based anticancer drug. Bioorganic chemistry. Bioorg Chem. 2019; 88:102925 doi: 10.1016/j.bioorg.2019.102925.

8. Farivar S, Malekshahabi T, Shiari R. Biological effects of low level laser therapy. J Lasers Med Sci. 2014;5(2):58-62.

9. Baxter GD, Liu L, Petrich S, Gisselman AS, Chapple C, Anders JJ, et al. Low level laser therapy (Photobiomodulation therapy) for breast cancer-related lymphedema: a systematic review. BMC Cancer. 2017;17(1):833. doi:org/10.1186/ s12885-017-3852-x.

10. Feller JF, Greenwood BM, Stafford RJ. Transrectal Laser Focal Therapy of Prostate Cancer. In: Imaging and Focal Therapy of Early Prostate Cancer [Internet]. Springer International Publishing; 2017.p. 325-43. doi: 10.1007/9783-319-49911-6_25.

11. Dougherty TJ. An update on photodynamic therapy applications. J Clin Laser Med Surg. 2002;20(1):3-7. doi: $10.1089 / 104454702753474931$.

12. Sawyers C. Targeted cancer therapy. Nature. 2004;432(7015):294-7. doi: 10.1038/nature03095.

13. Litwin MS, Tan H-J. The diagnosis and treatment of prostate cancer: a review. JAMA. 2017;317(24):2532-42. doi: 10.1001/jama.2017.7248.

14. Vale FA, Moreira MS, Almeida FCSd, Ramalho KM. Lowlevel laser therapy in the treatment of recurrent aphthous ulcers: a systematic review. Sci World J. 2015;2015:150412. doi:10.1155/2015/150412.

15. Mirza FN, Khatri KA. The use of lasers in the treatment of skin cancer: A review. J Cosmet Laser Ther. 2017;19(8):451458. doi: 10.1080/14764172.2017.1349321.

16. Bown SG. New techniques in laser therapy. BMJ.
1998;316(7133):754-7. doi: 10.1136/bmj.316.7133.754.

17. Fried NM. Therapeutic applications of lasers in urology: an update. Expert Rev Med Devices. 2006;3(1):81-94. doi:10.1586/17434440.3.1.81.

18. Galluzzi L, Senovilla L, Vitale I, Michels J, Martins I, Kepp $\mathrm{O}$, et al. Molecular mechanisms of cisplatin resistance. Oncogene. 2012;31(15):1869-83. doi:10.1038/onc.2011.384.

19. Nematbakhsh M, Ashrafi F, Pezeshki Z, Fatahi Z, Kianpoor F, Sanei M-H, et al. A histopathological study of nephrotoxicity, hepatoxicity or testicular toxicity: Which one is the first observation as side effect of Cisplatininduced toxicity in animal model? J Nephropathol. 2012;1(3):190. doi: 10.5812/nephropathol.8122.

20. Huang Y-Y, Chen AC-H, Hamblin M. Low-level laser therapy: an emerging clinical paradigm. SPIE Newsroom. 2009;9:1-3. doi: 10.1117/2.1200906.1669.

21. Gao X, Xing D. Molecular mechanisms of cell proliferation induced by low power laser irradiation. J Biomed Sci. 2009;16(1):4. doi: 10.1186/1423-0127-16-4.

22. Arnesano F, Losacco M, Natile G. An updated view of cisplatin transport. Eur J Inorg Chem. 2013;2013(15):270111. doi: 10.1002/ejic.201300001.

23. Kim CW, Lu JN, Go S-I, Jung JH, Yi SM, Jeong J-H, et al. p53 restoration can overcome cisplatin resistance through inhibition of Akt as well as induction of Bax. Int J Oncol Res. 2013;43(5):1495-502. doi: 10.3892/ijo.2013.2070.

24. Mansoori B, Mohammadi A, Doustvandi MA, Mohammadnejad F, Kamari F, Gjerstorff MF, et al. Photodynamic therapy for cancer: Role of natural products. Photodiagn. Photodiagn Photodyn Ther. 2019;26:395-404. doi: 10.1016/j.pdpdt.2019.04.033.

25. Crescenzi E, Varriale L, Iovino M, Chiaviello A, Veneziani BM, Palumbo G. Photodynamic therapy with indocyanine green complements and enhances low-dose cisplatin cytotoxicity in MCF-7 breast cancer cells. Mol Cancer Ther. 2004;3(5):537-44.

26. de Freitas LM, Soares CP, Fontana CR. Synergistic effect of photodynamic therapy and cisplatin: A novel approach for cervical cancer. J Photochem Photobiol B. 2014;140:365-73. doi: 10.1016/j.jphotobiol.2014.08.021. 